\title{
Inhibitory effect of Goreisan on vascular endothelial cell migration
}

\author{
Kazuhito Murakami, Ichiro Horie, Yoichiro Isohama
}

Lab. Appl. Pharmacol., Facul. of Pharm. Sci., Tokyo Univ. of Sci., Japan

\section{[Background]}

Goreisan is a Japanese Kampo medicine, which has been used for edema and headache. In recent years, the clinical studies indicated that Goreisan significantly suppresses the recurrence in patients of chronic subdural hematoma (CSDH). It has also been shown that, immature angiogenesis leads to destruction of blood vessels, following to hypertrophy of hematoma, recurrence or exacerbation. In this study, therefore, we hypothesized that the inhibitory effect of Goreisan on the recurrence of CSDH may be due to angiogenesis inhibition, and examined the effect of Goreisan on migration of vascular endothelial cells, which is the initial step of angiogenesis.

[Methods]

We treated Goreisan extract to human umbilical vascular endothelial cells (HUVEC) and measured migration activity by in vitro scratch assay. We checked protein expression of angiogenesis-related factors by western blotting.

[Results]

In HUVEC cells, Goreisan (0.01-0.1 mg/ml) attenuated vascular endothelial growth factor (VEGF)-induced migration in a dose-dependent manner. This effect was similar to that of MEK-ERK inhibitor, such as U0126. We then examined the effect of Goreian on ERK phosphorylation, and define that this herbal medicine inhibits VEGF-induced ERK phosphorylation. It was, therefore, suggested that Goreisan attenuated VEGF-induced cellular migration through ERK inhibition. Interestingly, Goreisan supressed steady state migration activity in the cells cultured without VEGF, although U0126 did not. We then focused on aquaporin-1 (AQP1), which is reported to be important in the direction of migration of endothelial cells. Goreisan considerably decreased AQP1 protein and mRNA expression in HUVEC treated with or without VEGF. The treatment of siRNA for AQP1 by itself suppressed steady state migration activity, and the suppressive effect of Goreisan on HUVEC migration was abolished in AQP1 knockdown cells. These results suggest that Goreisan can suppress steady state migration activity through the decrease in AQP1 expression.

[Conclusions]

These data indicated that Goreisan suppresses migration of endothelial cells through both inhibition of ERK and AQP1 expression, supporting the clinical preventive effect of Goreisan for CSDH recurrence. 